\title{
The application of hypergeometric functions to computing fractional order derivatives of sinusoidal functions
}

\author{
M. WŁODARCZYK and A. ZAWADZKI* \\ Faculty of Electrical Engineering, Automatic Control and Computer Science, Kielce University of Technology, \\ 7 Tysiąclecia Państwa Polskiego Ave., 25-314 Kielce, Poland
}

\begin{abstract}
In the paper, the analytical forms of fractional order derivatives of sinusoidal function according to definitions of Riemann Liouville and Caputo are presented. To determine the analytical form of the integrals appearing in definitions of derivatives of fractional order the Lommel functions from hypergeometrical functions family were applied. With the use of properties of the derivatives of fractional order - differential-integral there were presented the conception of generalized element of a single equation, which depending on the value of the derivative order, can be inductor, resistor, capacitor, or a hypothetical element of a fractional order differential equation.
\end{abstract}

Key words: fractional degree derivative; hypergeometric function; Lommel function.

\section{Introduction}

Fractional order derivatives, also known as differentialintegrals are applied in ever new areas of knowledge, such as:

- the modeling of the behavior of polymers and highly elastic materials [1];

- the examination of the phenomena of relaxation of organic dielectrics. [2];

- automatics, $P i^{\lambda} D^{\mu}$ controllers [3-5];

- modeling of electrochemical processes [6];

- modeling of traffic in computer networks [7];

- supercapacitors modeling $[8,9]$.

In this paper, we attempt to apply fractional order derivatives in circuit theory.

It is assumed that there exist hypothetical components (quasi-capacitative, quasi-inductive), in which the connections between currents and voltages or voltages and currents are defined using a fractional order derivative, for example:

$$
i_{C}=C \frac{d^{\alpha} u_{C}}{d t^{\alpha}}, \quad u_{L}=L \frac{d^{\alpha} i_{L}}{d t^{\alpha}},
$$

which can, in general, be written as a relation between the forcing - input signal $x$ and the output signal - response $y$

$$
y=B \frac{d^{\alpha} x}{d t^{\alpha}},
$$

where $B$ denotes a constant numeric coefficient.

There are many indications that the real elements that meet such equations are: supercapacitors [9], coils with an open ferromagnetic core [10] and non-linear elements with the ambiguous characteristics.

The cases where the input signal is a unit step were considered in [11]. It would be interesting to determine the response of these elements to sinusoidal inputs. Therefore, the task is to compute:

$$
\frac{d^{\alpha}}{d t^{\alpha}} A \sin (\omega t)
$$

Numerical algorithms for computing such derivatives were given in $[12,13]$ but an analytic solution is not known.

The aim of this work is to find an analytical form of the derivative (3) basing on two known definitions of fractional order derivatives - a derivative according to definitions of Riemann-Liouville and Caputo. To determine the solution, the Lommel functions belonging to the family of hypergeometric functions were used.

\section{Derivatives of sinusoidal functions according to the definition of Riemann-Liouville}

Using the definition of Riemann-Liouville (R-L) [14-17]:

$$
{ }_{a} D_{t}^{\alpha} f(t)=\frac{1}{\Gamma(k-\alpha)} \frac{d^{k}}{d t^{k}} \int_{a}^{t}(t-\tau)^{k-\alpha-1} f(\tau) d \tau,
$$

where $k-1 \leq \alpha \leq k, \boldsymbol{\Gamma}(\alpha)-$ gamma (Euler) function, we get the response of the system for the fractional derivative of order $0<\alpha<1$ in the form:

$$
y=B_{0} D_{t}^{\alpha} x=\frac{A B}{\Gamma(1-\alpha)} \frac{d}{d t} \int_{0}^{t}(t-\tau)^{-\alpha} \sin (\omega \tau) d \tau .
$$

As can be seen, the most difficult task is to compute the definite integral analytically, as in the next step its derivative should be calculated. This integral can be determined using Lommel's function from the family of hypergeometric functions.

\footnotetext{
*e-mail: a.zawadzki@tu.kielce.pl
} 
Hypergeometric functions are an extensive class of functions, whose characteristic feature is that their Tschebyschev coefficients can be expressed sufficiently simply by values of functions from this class [18]. Hence, the integer from relation (5) is expressed as follows:

$$
\int_{0}^{t}(t-\tau)^{-\alpha} \sin (\omega \tau) d \tau=\frac{(\omega t \alpha L 1-L 2) \omega^{\alpha-\frac{3}{2}} \cdot t^{-\frac{1}{2}}}{(\alpha-1)}
$$

where $L 1=$ LommelS $1(1 / 2-\alpha, 3 / 2, \omega t), L 2=$ LommelS $1(3 / 2-\alpha, 1 / 2, \omega t)$.

The Lommel function is defined in the following way [18]:

$$
\begin{gathered}
\operatorname{Lommel} S 1(\mu, \nu, x)=s_{\mu \nu}(x) \\
=\frac{x^{\mu+1}}{(\mu-\nu+1)(\mu+\nu+1)} \\
\cdot{ }_{2} F_{1}\left(1, \frac{1}{2}(\mu-\nu+3), \frac{1}{2}(\mu+\nu+3),-\frac{1}{4} x^{2}\right),
\end{gathered}
$$

${ }_{2} F_{1}(\alpha, \beta, \gamma, x)$ is a hypergeometric function defined as [18]:

$$
{ }_{2} F_{1}(\alpha, \beta, \gamma, x)=\sum_{k=0}^{\infty} \frac{(\alpha)_{k}(\beta)_{k}}{(\gamma)_{k} k !} x^{k},
$$

where $(a)_{k}$ is Pochhammer's symbol expressed by the formula:

$$
(a)_{k}=a(a+1) \ldots(a+k-1) \quad \text { for } \quad(a)_{0}=1 .
$$

Therefore, functions denoted as $L 1$ and $L 2$ obtain the form:

$$
\begin{gathered}
L 1=\frac{(\omega t)^{1.5-\alpha}}{(-\alpha)(3-\alpha)} \sum_{k=0}^{\infty}(-1)^{k} \frac{(1)_{k}(1-0.5 \alpha)_{k}}{(2.5-0.5 \alpha)_{k} 4^{k} k !}(\omega t)^{2 k}, \\
L 2=\frac{(\omega t)^{2.5-\alpha}}{(2-\alpha)(3-\alpha)} \sum_{k=0}^{\infty}(-1)^{k} \frac{(1)_{k}(2-0.5 \alpha)_{k}}{(2.5-0.5 \alpha)_{k} 4^{k} k !}(\omega t)^{2 k} .
\end{gathered}
$$

Whereas the integral (6) is expressed by the formula;

$$
\begin{gathered}
\int_{0}^{t}(t-\tau)^{-\alpha} \sin (\omega \tau) d \tau=\frac{-\omega t^{2-\alpha}}{(\alpha-1) \cdot(3-\alpha)} \\
\left(\sum_{k=0}^{\infty}\left\{\frac{(-1)^{k}(1)_{k}(\omega t)^{2 k}}{4^{k}(2.5-0.5 \alpha)_{k} k !}\left[(1-0.5 \alpha)_{k}+\frac{(2-0.5 \alpha)_{k}}{(2-\alpha)}\right]\right\}\right),
\end{gathered}
$$

or in the form suitable for differentiation with respect to the variable $t$ :

$$
\begin{gathered}
\int_{0}^{t}(t-\tau)^{-\alpha} \sin (\omega \tau) d \tau=\frac{-\omega}{(\alpha-1) \cdot(3-\alpha)} \\
\cdot\left(\sum_{k=0}^{\infty}\left\{\frac{(-1)^{k}(1)_{k} \omega^{2 k} t^{2-\alpha+2 k}}{4^{k}(2.5-0.5 \alpha)_{k} k !}\left[(1-0.5 \alpha)_{k}+\frac{(2-0.5 \alpha)_{k}}{(2-\alpha)}\right]\right\}\right) .
\end{gathered}
$$

To determine the derivative of the integral (6) with respect to time, the definite series defined in (12) should be differentiated term by term. One can proceed in such a way only if every term of the series is continuous for a finite value of the variable, with respect to which one is differentiating (in this case, this it the variable $t$ ) [19].
Hence the derivative equals:

$$
\begin{gathered}
\frac{d}{d t} \int_{0}^{t}(t-\tau)^{-\alpha} \sin (\omega \tau) d \tau=\frac{-\omega}{(\alpha-1) \cdot(3-\alpha)} \\
\left(\sum_{k=0}^{\infty}\left\{\begin{array}{c}
\frac{(-1)^{k}(1)_{k} \omega^{2 k}(2-\alpha+2 k) t^{1-\alpha+2 k}}{4^{k}(2.5-0.5 \alpha)_{k} k !} \\
\cdot\left[(1-0.5 \alpha)_{k}+\frac{(2-0.5 \alpha)_{k}}{(2-\alpha)}\right]
\end{array}\right\}\right)
\end{gathered}
$$

The responses for sinusoidal input to the system (5) for fractional derivative of order $0<\alpha<1$ according to the Riemann-Liouville definition have the analytic form:

$$
\begin{gathered}
y=\frac{-A B \omega}{(\alpha-1) \cdot(3-\alpha) \cdot \boldsymbol{\Gamma}(1-\alpha)} \\
\cdot \sum_{k=0}^{\infty}\left\{\begin{array}{c}
{\left[(1-0.5 \alpha)_{k}+\frac{(2-0.5 \alpha)_{k}}{(2-\alpha)}\right]} \\
\frac{(-1)^{k}(2-\alpha+2 k)(1)_{k} \omega^{2 k} t^{1-\alpha+2 k}}{4^{k}(2.5-0.5 \alpha)_{k} k !}
\end{array}\right\} .
\end{gathered}
$$

Considering fractional Riemanna-Liouville derivatives of order $-1<\alpha<0$ we obtain:

$$
{ }_{0} D_{t}^{\alpha} f(t)=\frac{1}{\Gamma(-\alpha)} \int_{0}^{t}(t-\tau)^{-\alpha-1} f(\tau) d \tau .
$$

The integral in formula (15) can be expressed in an analytic form:

$$
\begin{gathered}
\int_{0}^{t}(t-\tau)^{-\alpha-1} f(\tau) d \tau \\
=\frac{-t^{2} \omega t^{-\alpha-1}\left(-1+(\omega t)^{\alpha-0.5} L 2\right)}{\alpha(\alpha-1)},
\end{gathered}
$$

where $L 2$ denotes the Lommel function defined by formula (10).

Therefore, responses for sinusoidal inputs (5) to the system for a fractional derivative of order $-1<\alpha<0$ according to the definition of Riemann-Liouville have the form:

$$
\begin{gathered}
y=\frac{\omega A B}{\alpha(\alpha-1) \boldsymbol{\Gamma}(-\alpha)} \\
\left(\begin{array}{c}
t^{1-\alpha}-\frac{1}{(2-\alpha)(3-\alpha)} \\
\cdot \sum_{k=0}^{\infty}(-1)^{k} \frac{(1)_{k}(2-0.5 \alpha)_{k}(\omega)^{2 k+2}(t)^{2 k+3-\alpha}}{4^{k}(2.5-0.5 \alpha)_{k} k !}
\end{array}\right),
\end{gathered}
$$

or in an expanded form:

$$
\begin{gathered}
y=\frac{\omega A B}{\alpha(\alpha-1) \boldsymbol{\Gamma}(-\alpha)} \\
\left(\begin{array}{c}
t^{1-\alpha}-\frac{1}{(2-\alpha)(3-\alpha)} \\
\cdot \sum_{k=0}^{\infty} \frac{(1)_{k}(2-0.5 \alpha)_{k}(\omega)^{2 k+2}(t)^{2 k+3-\alpha}}{4^{k}(2.5-0.5 \alpha)_{k} k !}
\end{array}\right) .
\end{gathered}
$$


The application of hypergeometric functions to computing fractional order derivatives of sinusoidal functions

Considering the fractional order Riemann-Liouville derivatives of orders from the interval $1<\alpha<2$ one obtains the expression:

$$
{ }_{0} D_{t}^{\alpha} f(t)=\frac{1}{\Gamma(2-\alpha)} \frac{d^{2}}{d t^{2}} \int_{0}^{t}(t-\tau)^{1-\alpha} f(\tau) d \tau .
$$

The integral in the formula (15) can be expressed in an analytic form:

$$
\int_{0}^{t}(t-\tau)^{1-\alpha} f(\tau) d \tau=\omega^{\alpha-1.5} t^{0.5} L 2 .
$$

Computing its second derivative, one obtains:

$$
\begin{gathered}
\frac{d^{2}}{d t^{2}} \int_{0}^{t}(t-\tau)^{1-\alpha} f(\tau) d \tau=\frac{\omega}{(2-\alpha) \cdot(3-\alpha)} \\
\sum_{k=0}^{\infty}\left[\frac{(-1)^{k}(1)_{k}(2-0.5 \alpha)_{k}(3-\alpha+2 k) \cdot(2-\alpha+2 k)}{(2.5-0.5 \alpha)_{k}}\right. \\
\left.\cdot \frac{\omega^{2 k} t^{1-\alpha+2 k}}{4^{k} k !}\right] .
\end{gathered}
$$

Hence, the responses for sinusoidal imput (5) to the system for fractional derivative of order $1<\alpha<2$ according to the definition of Riemann-Liouville obtain the analytic form:

$$
\begin{gathered}
y=\frac{A B \omega}{(2-\alpha) \cdot(3-\alpha) \cdot \boldsymbol{\Gamma}(2-\alpha)} \\
\cdot \sum_{k=0}^{\infty}\left[\frac{(-1)^{k}(1)_{k}(2-0.5 \alpha)_{k}(3-\alpha+2 k) \cdot(2-\alpha+2 k)}{(2.5-0.5 \alpha)_{k}}\right. \\
\left.\cdot \frac{\omega^{2 k} t^{1-\alpha+2 k}}{4^{k} k !}\right] .
\end{gathered}
$$

Analogously, one can obtain analytic formulas for a fractional order from any interval.

\section{Derivatives of sinusoidal functions according to the definition of Caputo}

Another definition of a fractional order derivative is the definition of Caputo [14, 15, 20-25]:

$$
\begin{gathered}
{ }_{a}^{C} D_{t}^{\alpha} f(t)=\frac{1}{\Gamma(\alpha-n)} \int_{a}^{t} \frac{f^{(n)}(\tau)}{(t-\tau)^{\alpha+1-n}} d \tau, \\
n-1 \leq \alpha \leq n .
\end{gathered}
$$

As can be seen, in this case the calculation will be easier to do, since the derivatives of order $n$ are under the integral and concern the sine function. Hence, for fractional orders $0<\alpha<1$ one obtains:

$$
\begin{aligned}
& { }_{a}^{C} D_{t}^{\alpha} f(t)=\frac{1}{\Gamma(1-\alpha)} \int_{0}^{t} \frac{f^{(1)}(\tau)}{(t-\tau)^{\alpha}} d \tau \\
& =\frac{\omega}{\Gamma(1-\alpha)} \int_{0}^{t}(t-\tau)^{-\alpha} \cos (\omega \tau) d \tau .
\end{aligned}
$$

The analytic form of the integral in formula (21) is shown by the expression:

$$
\int_{0}^{t}(t-\tau)^{-\alpha} \cos (\omega \tau) d \tau=\frac{t^{1-\alpha}\left[-1+(\omega t)^{\alpha-0.5} L 2\right]}{(\alpha-1)} .
$$

Whereas the responses for sinusoidal inputs (5) to the system for a fractional derivative of order $0<\alpha<1$ according to the Caputo definition obtain the form:

$$
\left.\begin{array}{c}
y=\frac{\omega A B t^{1-\alpha}}{(\alpha-1) \boldsymbol{\Gamma}(1-\alpha)} \\
-1+\frac{1}{(2-\alpha) \cdot(3-\alpha)} \\
\cdot \sum_{k=0}^{\infty}(-1)^{k} \frac{(1)_{k}(2-0.5 \alpha)_{k}}{(2.5-0.5 \alpha)_{k} 4^{k} k !}(\omega t)^{2 k+2}
\end{array}\right]
$$

and in an expanded form.

$$
\begin{gathered}
y=\frac{\omega A B t^{1-\alpha}}{(\alpha-1) \boldsymbol{\Gamma}(1-\alpha)} \\
{\left[\begin{array}{c}
-1+\frac{1}{(2-\alpha) \cdot(3-\alpha)} \\
\cdot \sum_{k=0}^{\infty} \frac{(1)_{k}(2-0.5 \alpha)_{k}(\omega t)^{2 k+2}}{4^{k}(2.5-0.5 \alpha)_{k} k !}
\end{array}\right]}
\end{gathered}
$$

For a fractional derivative of order $-1<\alpha<0$ according to the definition of Caputo we obtain, in sequence:

$$
\begin{aligned}
& { }_{0}^{C} D_{t}^{\alpha} f(t)=\frac{1}{\Gamma(-\alpha)} \int_{a}^{t} \frac{f^{(0)}(\tau)}{(t-\tau)^{\alpha+1}} d \tau \\
& =\frac{1}{\Gamma(-\alpha)} \int_{a}^{t}(t-\tau)^{-\alpha-1} \sin (\omega \tau) d \tau .
\end{aligned}
$$

The integral in the above formula is:

$$
\begin{gathered}
\int_{0}^{t}(t-\tau)^{-\alpha-1} \sin (\omega \tau) d \tau \\
=\frac{-\omega t^{-\alpha+1}\left[-1+(\omega t)^{\alpha-0.5} L 2\right]}{\alpha(\alpha-1)} .
\end{gathered}
$$

Substituting (27) into Eq. (5) we get:

$$
\begin{gathered}
y=\frac{\omega A B t^{-\alpha+1}}{\alpha(\alpha-1) \Gamma(-\alpha)} \\
{\left[\begin{array}{c}
-1+\frac{(\omega t)^{2}}{(2-\alpha) \cdot(3-\alpha)} \\
\cdot \sum_{k=0}^{\infty}(-1)^{k} \frac{(1)_{k}(2-0.5 \alpha)_{k}}{(2.5-0.5 \alpha)_{k} 4^{k} k !}(\omega t)^{2 k}
\end{array}\right]}
\end{gathered}
$$


and, taking into account the Lommel function (L2):

$$
\begin{gathered}
y=\frac{\omega A B t^{-\alpha+1}}{\alpha(\alpha-1) \Gamma(-\alpha)} \\
{\left[\begin{array}{c}
1-\frac{1}{(2-\alpha)(3-\alpha)} \\
\cdot \sum_{k=0}^{\infty} \frac{(1)_{k}(2-0.5 \alpha)_{k}(\omega t)^{2 k+2}}{4^{k}(2.5-0.5 \alpha)_{k} k !}
\end{array}\right]}
\end{gathered}
$$

Whereas for the fractional derivative of order $1<\alpha<2$ according to the definition of Caputo we obtain:

$$
\begin{aligned}
& { }_{0}^{C} D_{t}^{\alpha} f(t)=\frac{1}{\Gamma(2-\alpha)} \int_{0}^{t} \frac{f^{(2)}(\tau)}{(t-\tau)^{\alpha-1}} d \tau \\
& =\frac{-\omega^{2}}{\Gamma(2-\alpha)} \int_{0}^{t}(t-\tau)^{-\alpha+1} \sin (\omega \tau) d \tau,
\end{aligned}
$$

where the integral in (29) is expressed as follows:

$$
\int_{0}^{t}(t-\tau)^{-\alpha+1} \sin (\omega \tau) d \tau=\omega^{\alpha-1.5} t^{0.5} L 2 .
$$

As a result, the system response for the fractional derivative represented by formula (5) is:

$$
\begin{gathered}
y=\frac{-\omega^{2} A B}{\Gamma(2-\alpha)} \frac{t^{3-\alpha}}{(2-\alpha) \cdot(3-\alpha)} \\
\cdot \sum_{k=0}^{\infty}(-1)^{k} \frac{(1)_{k}(2-0.5 \alpha)_{k}}{(2.5-0.5 \alpha)_{k} 4^{k} k !}(\omega t)^{2 k}
\end{gathered}
$$

and with regard to the Lommel function (L2) Eq. (31a) becomes:

$$
\begin{gathered}
y=\frac{-\omega^{\alpha-1} A B}{(2-\alpha)(3-\alpha) \boldsymbol{\Gamma}(2-\alpha)} \\
\sum_{k=0}^{\infty} \frac{(1)_{k}(2-0.5 \alpha)_{k}(\omega t)^{2 k+3-\alpha}}{4^{k}(2.5-0.5 \alpha)_{k} k !} .
\end{gathered}
$$

Analogously, one can compute the analytic forms of fractional derivatives from any interval $k-1<\alpha<k$ for any integer $k$.

\section{Numeric realization}

The numerical realization of fractional order derivatives of sinusoidal functions described analytically - according to Riemann-Liouville definition - Eqs. (14), (17a) and (21) and according to Caputo definition - (25a), (28a) and (31a) is not simple because these equations present infinite sums of alternating series. Ingredients successively alter their signs - factor $(-1)^{k}$, and it's difficult to determine how many elements should be summed. In addition, a steadily growing exponent $t$ makes, that for large times the series is divergent. However, by using appropriate scaling and selection of the amounts of the components, the convergence can be achieved. Figure 1 shows a comparison of derivatives of 0.9 order calculated according to Riemann-Liouville and Caputo definitions with the integer derivative of the first order i.e. the cosinus function.

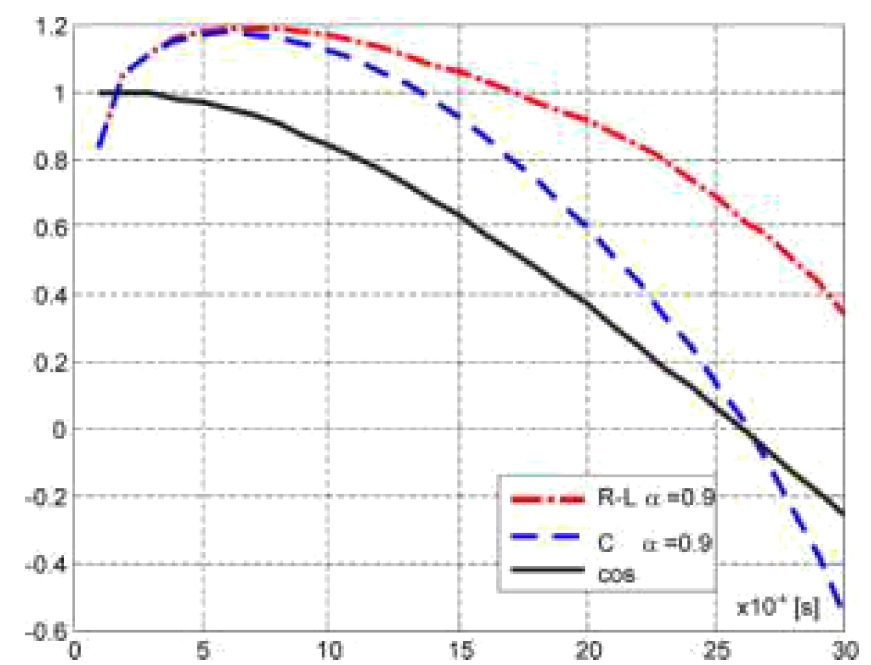

Fig. 1. Comparison of derivatives of 0.9 order and integer derivative of the first order

In analyzing these waveforms it can be seen that for small values of time $t$ fractional order derivatives calculated according to Riemann-Liouville and Caputo definitions - overlap while with increasing $t$ - they show increasing differences. It should also be pointed out, that only derivative according to Caputo definition has zero in the same place as the cosinus function.

Next figures show the waveforms of fractional derivatives of $0.9,0.8$ and 0.7 order, calculated according to the RiemannLiouville (Fig. 2) and Caputo (Fig. 3) definitions. As can be seen, in both cases decreasing of the derivative's order causes a "flattening" of the waveform.

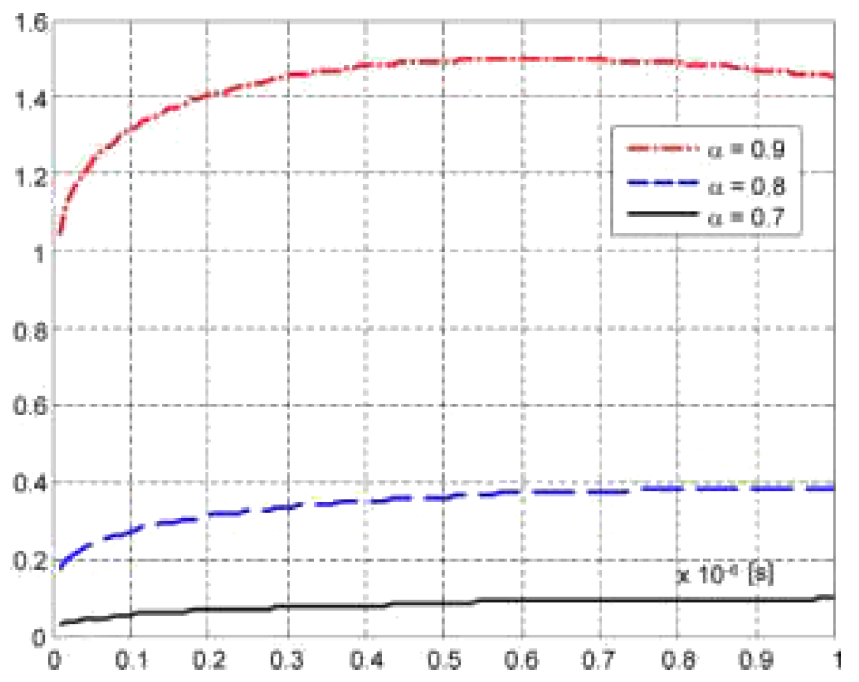

Fig. 2. Fractional order derivatives calculated according to RiemannLiouville definition 


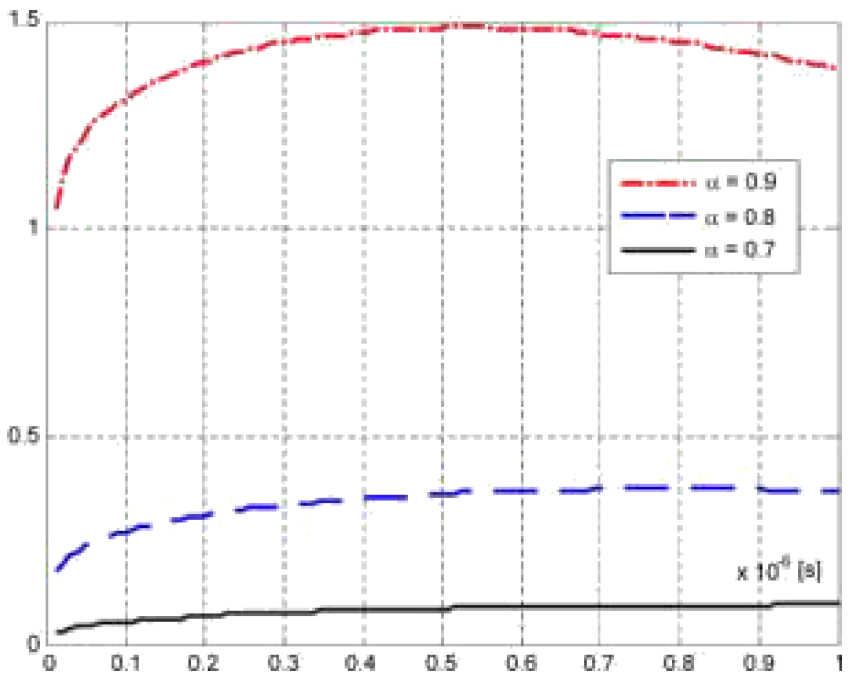

Fig. 3. Fractional order derivatives calculated according to Caputo definition

\section{Summary}

In this paper, we outlined analytic forms of the fractional order derivatives of a sinusoidal function according to the definition of Riemann-Liouville and Caputo. We used the Lommel function from the family of hypergeometric functions.

These functions are expressed in the form of power series with respect to the variable $t$, which is important inasmuch as it is straightforward to differentiate and integrate them and therefore one can easily determine a fractional derivative of a sinusoidal function for any interval $k-1<\alpha<k$ for any integer $k$, according to both the definition of RiemannLiouville and Caputo.

Using the properties of the derivative of fractional order - the differential-integral, one can introduce the concept of a generalized component given by the equation:

$$
u=B \frac{d^{\alpha} i}{d t^{\alpha}}
$$

Depending on the value for the order of the derivative $\alpha$ :

- for $\alpha=0-$ it would be a resistor with resistance $R=B$,

- for $\alpha=1-$ it would be an ideal inductor with inductance $L=B$,

- for $\alpha=-1$ - it would be an ideal capacitor with capacity $C=1 / B$.

For other values of order $\alpha$, it would be a component whose properties have not been defined yet.

The numerical realization of fractional order derivatives of sinusoidal functions described analytically - according to Riemann-Liouville definition and according to Caputo definition is not simple, however, by using appropriate scaling and selection of the amounts of the components, the convergence can be achieved.

\section{REFERENCES}

[1] R.L. Bagley, "Fractional calculus - a different approach to the analysis of viscoelastically damped structures", AIAA J. 21, 741-748 (1983).

[2] M.E. Reyes-Melo, J.J. Martinez-Vega, C.A. Guerrero-Salazar, and U. Ortiz-Mendes, "Modelling of relaxation phenomena in organic dielectric materials, application of differential and integral operators of fractional order", J. Optoelectronics and Advanced Matrials 6 (3), 1037-1043 (2004).

[3] T. Kaczorek, Positive ID and 2D Systems, Springer Verlag, London, 2002.

[4] D. Sierociuk, "Estimation and control of discrete dynamical systems of fractional order described in a state space", Doctoral Dissertation, Warsaw University of Technology, Warsaw, 2007, (in Polish).

[5] A. Ruszewski, "Stability regions of closed loop system with time delay inertial plant of fractional order and fractional order PI controller", Bull. Pol. Ac.: Tech. 56 (4), 329-332 (2008).

[6] T. Kosztołowicz, The Use of Differential Equations with Fractional Derivatives to Describe a Subdiffusion, Jan Kochanowski University in Kielce, Kielce, 2008, (in Polish).

[7] V. Zaborovsky and R. Meylanov, "Informational network traffic model based on fractional calculus", Proc. Int. Conf. Info-tech and Info-net 1, CD-ROM (2001).

[8] A. Dzieliński, D. Sierociuk, and G. Sarwas, "Some applications of fractional order calculus", Bull. Pol. Ac.: Tech. 58 (4), 583-592 (2010).

[9] M. Włodarczyk and A. Zawadzki, "Connecting a capacitor to direct voltage in aspect of fractional degree derivatives", Przeglą Elektrotechniczny 10, 120-123 (2009).

[10] A. Zawadzki, "Applying derivatives of the fractional order for modeling transient states in electrical circuits containing inductance", Przegląd Elektrotechniczny 4, 92-94 (2013), (in Polish).

[11] M. Włodarczyk and A. Zawadzki, "Algorithms of calculations of fractional derivatives of unit function", in: Information Technology in Science, Technology and Education, pp. 87-90, Scientific Publishing House of Institute for Sustainable Technologies-National Research Institute, Radom 2009, (in Polish).

[12] D. Brzeziński and P. Ostalczyk, "Numerical evaluation of fractional differ-integrals of some periodical functions via the IMT transformation”, Bull. Pol. Ac.: Tech. 60 (2), 285-292 (2012).

[13] A. Zawadzki and M. Włodarczyk, "The numerical calculations of fractional order derivatives for sinusoidal functions", in: Science, Technology, Education and Modern Information Technology, pp. 194-205, Scientific Publishing House of Institute for Sustainable Technologies-National Research Institute, Radom 2011, (in Polish).

[14] I. Podlubny, Fractional Differential Equations, Academic Press, San Diego, 1999.

[15] P. Ostalczyk, The Outline of Differential and Integral Calculus of Fractional Order. The Theory and Applications in Automatic Control, Publishing Department of Technical University of Łódź, Łódź, 2008, (in Polish).

[16] T. Kaczorek, "Fractional positive linear system and electrical circuits", Przeglą Elektrotechniczny 9, 135-141 (2008).

[17] D.W. Brzeziński and P. Ostalczyk, "High-accuracy numerical integration methods for fractional order derivatives and integrals computations", Bull. Pol. Ac.: Tech. 62 (4), 723-733 (2014). 


\section{Włodarczyk and A. Zawadzki}

[18] S. Paszkowski, Numerical Applications of Chebyshev Polynomials and Series, PWN, Warszawa, 1975, (in Polish).

[19] N.W. McLachlan, Bessel Functions for Engineers, Clarendon Press, Oxford, 1955.

[20] M. Caputo, Elasticilà c Dissipazione, Zanichelli, Bologna, 1969.

[21] K.B. Oldham and J. Spanier, The Fractional Calculus, Academic Press, New York, 1974.

[22] T. Kaczorek., Selected Problems of Fractional Systems Theory, Springer-Verlag, Berlin-Heidelberg, 2011.
[23] T. Kaczorek, "Analysis of fractional linear electrical circuits in transient states", Przegląd Elektrotechniczny 6, 191-195 (2010), (in Polish)

[24] M. Busłowicz, "Stability analysis of continuous-time linear systems consisting of $n$ subsystems with different fractional orders", Bull. Pol. Ac.: Tech. 60 (2), 279-284 (2012).

[25] T. Kaczorek, "Reduced-order fractional descriptor observers for fractional descriptor continuous-time linear system", Bull. Pol. Ac.: Tech. 62 (4), 889-895 (2014). 